\title{
449.
}

\section{SKETCH OF RECENT RESEARCHES UPON QUARTIC AND QUINTIC SURFACES.}

[From the Proceedings of the London Mathematical Society, vol. III. (1869-1871), pp. 186-195. Read Nov. 10, 1870.]

THE classification of quartic surfaces is even as to its highest divisions incomplete; and it is by no means easy to make it at once exhaustive and precise; an enumeration of all the primâ facie possible cases would include forms which do not really exist. Thus the singular curve (if any) is of the order 1, 2, or 3-but in the case where the order is $=3$, the curve, as is at once evident, cannot be a plane cubic, nor (among other excluded forms) a system of three non-intersecting lines. And certain forms of the singular curve, e.g. all but one of the admissible forms of a curve of the order 3, make the surface to be a scroll, so that, if (as is convenient) we wish to separate the scrolls, certain forms otherwise admissible must be excluded. The expression "singular" means double or cuspidal, or refers to a higher singularity, but the cases of higher singularity are very special. I will, at the cost of some inaccuracy, use the expression "nodal" as meaning, in general, double, but as including the signification "cuspidal"; and, if there are any cases of higher singularity, as extending to cases of higher singularity: and I provisionally arrange the non-scrolar quartic surfaces as follows:

1. Without a nodal curve.

2. With a nodal line.

3. With a nodal conic, or line-pair (pair of intersecting lines).

4. With three nodal lines (not in the same plane) meeting in a point.

(Observe that the omitted cases are cases which, as I believe, ought to be omitted; thus the case of a nodal skew cubic is omitted, because the surface is then of necessity a scroll.) And to these I join:

5. The quartic scrolls;

omitting altogether the torse and cones. 
The references, by the name of the author, and number (if any) of his paper, are to the subjoined list of Memoirs.

As to the scrolls, we have Cayley (3) and (4), and Cremona; the division into 12 species is, I believe, complete: see post, the remarks upon Schwarz's paper on quintic scrolls.

As regards the non-scrolar surfaces:

1. Without a singular curve. The surface may be without a cnicnode (conical point), or it may have any number of cnicriodes up to 16, Cayley (7): the cases of singularity higher than a cnicnode are probably very numerous, but they have been scarcely at all examined. The memoir just referred to relates chiefly to the several cases of not more than 10 nodes; the cases of 11,12,13,14, 15, 16 nodes are considered incidentally, Kummer (2), but it was not the object of his paper to make an enumeration, and there may be cases which are not considered; the discussion of the cases considered is very full and interesting. The case of 16 nodes is also considered, Kummer (1). As to the surface with 16 nodes, it is to be remarked that the wavesurface, or generally the surface obtained by the homographic deformation of the wavesurface-called, Cayley (1), the "tetrahedroid"-is a special form of surface with 16 nodes: its relation to the general surface is explained, Cayley (2).

2. Quartic surface with nodal line: considered incidentally, Clebsch (2) and (3). There are through the nodal line 8 planes, each meeting the surface in a line-pair: considering any 7 of these, and taking out of each of them a line, the 7 lines are met by a conic which also meets a determinate line out of the remaining line-pair; there are thus on the surface $2^{7},=128$, conics; viz., these form 64 pairs, each pair lying in a plane, and being the complete intersection of the surface by such plane; the number of these planes is of course $=64$.

Although not properly included in the present case, I mention the quartic surface which is the reciprocal of the cubic surface XIX $=12-B_{6}-C_{2}$, Cayley (5): the nodal curve is here an oscnodal line counting as three nodal lines.

3. Quartic surfaces with nodal conic. Such a surface may be without cnicnodes, or it may have $1,2,3$, or 4 cnicnodes; the cases, other than that of 3 cnicnodes, are mentioned, Kummer (3); but the question is examined, and the remaining case of 3 cnicnodes established, Cayley (6).

The general case of the nodal conic without cnicnodes is elaborately considered, Clebsch (1): it is shown that there are on the surface 16 lines, each meeting the conic, and which in their arrangement are strikingly analogous to the 27 lines on a cubic surface; viz., if on a cubic surface we select at pleasure any one of the 27 lines, and through this line draw a plane which besides meets the cubic surface in a conic; then, disregarding the line in question and the 10 lines which meet it, the remaining 16 lines each meet the conic, and are related to it and to each other in the same manner that the 16 lines of the quartic surface are related to the nodal 
conic and to each other. And the ground hereof appears, Geiser; viz., it is shown that the quartic surface with the nodal conic, is rationally transformable into a cubic surface, the 16 lines and the nodal conic corresponding respectively to the 16 lines and the conic of the cubic surface.

The several cases of $1,2,3$, and 4 cnicnodes are considered, Korndörfer.

In the case where the nodal conic is the circle at infinity, the surfaces have been termed "anallagmatic" (perhaps "bicircular" would be a more convenient name), and a great deal has been written upon these surfaces by Moutard, Clifford, and others. Such a surface may of course have 1, 2, 3, or 4 cnicnodes; these surfaces, viz. the cnicnodal anallagmatics, in fact arise from the inversion of a quadric surface by the method of reciprocal radius vectors (that is, by the change of $x, y, z$ into $\left.\frac{x}{r^{2}}, \frac{y}{r^{2}}, \frac{z}{r^{2}}\right)$ : the centre of inversion is a node on the quartic surface. If the quadric surface is a cone, there is another node, the inverse point of the vertex; if the quadric surface is one of revolution, there are two other nodes; and if it is a cone of revolution, there are three other nodes-viz., in all, four nodes. The last-mentioned surface is, or includes, the Cyclide; viz., this is a quartic surface having the circle at infinity for a nodal curve, and having besides four nodes, which are a system of skew antipoints. The surface was first considered by Dupin (Applications de Géométrie \&c., 1822) as the envelope of a sphere touching three given spheres-its lines of curvature are thus circles; and the surface has been very frequently considered in refẹrence to this property and otherwise: see Maxwell, where a classification (not quite complete) is made of the different forms of the surface, and also stereographic drawings given. It is to be observed, that one interesting form, the parabolic cyclide, is not a quartic but a cubic surface.

In the class of surfaces which have been under consideration, the cnicnodes have been points not on the nodal conic-in fact, a point on a nodal curve cannot be, properly speaking, a cnicnode, though it may be a point of higher singularity in the nature of a cnicnode; viz., there may be on the nodal curve points which, in the classification of the surfaces, must be counted as cnicnodes. Such a case presents itself in the "Conic Torus," or surface generated by the rotation of a conic about a line whether not in or in the plane of the conic. The surface has been considered, De la Gournerie (1), although more in reference to the constructions of descriptive geometry than as a theory of pure geometry, and Cayley (6). The surface has a nodal circle, and upon it two singular points, the circular points at infinity; so that it belongs to the case of a nodal conic with two cnicnodes. In the particular case where the axis of rotation is in the plane of the conic, then there are on the axis two chicnodes; so that the case is that of a nodal conic and four cnicnodes; and when the generating conic is a circle, viz., when the surface is the ordinary torus, or anchor ring, generated by the rotation of a circle about a line in its own plane, then the nodal conic is the circle at infinity having upon it two cnicnodal points (its intersections by the planes at right angles to the axis) and the surface has also two cnicnodes on the axis: the surface, although presenting considerable peculiarity, may be 
regarded as a particular case of the cyclide. In reference to the plane sections of the conic torus and its various particular cases, see De la Gournerie (2); the ordinary torus has been the subject of numerous papers by Darboux and others, and possesses very interesting properties.

In connexion with the foregoing, I speak of the surfaces having a cuspidal conic: the general case is briefly referred to, Cayley (6); viz., this is the surface $(A A)$ the equation of which is $V^{2}-x^{3} y=0$, and which it is shown has a reciprocal of the order 6 . A special case is the surface $(A B)$ having a reciprocal of the order 3 ; viz., the quartic surface is here the reciprocal of the cubic surface $\mathrm{XX}=12-U_{8}$, Cayley (5). And it appears from the memoir rast referred to, that there is another cubic surface, $\mathrm{XVII}=12-2 B_{3}-C_{2}$, the reciprocal of which is a quartic surface having a cuspidal conic. But the theory of the quartic surfaces with a cuspidal conic has been hardly at all considered.

I do not know that anything has been done in regard to the quartic surfaces where the nodal conic becomes a line-pair; that is, where we have two intersecting nodal lines. Although not properly, belonging to the case in question, I mention here the quartic surface which is the reciprocal of the cubic surface XVIII $=12-B_{4}-2 C_{2}$, Cayley (5); the nodal curve consists of two intersecting lines, but one of them is tacnodal, counting as two nodal lines.

4. Quartic surface with three nodal lines (not in the same plane) meeting in a point. This is, in fact, Steiner's quartic surface; and it has been the subject of numerous investigations.

The equation of the surface may be taken to be $\sqrt{ } x+\sqrt{ } y+\sqrt{ } z+\sqrt{ } w=0$; and the surface thus presents itself as the reciprocal of the cubic surface $\frac{1}{x}+\frac{1}{y}+\frac{1}{z}+\frac{1}{w}=0$, $\left(\mathrm{XVI}=12-4 C_{2}\right.$, ) with four cnicnodes $\left({ }^{1}\right)$.

It was convenient to make the foregoing enumeration before speaking of Kummer's paper (3), and of the several memoirs which relate to the Abbildung of certain quartic and quintic surfaces.

As regards Kummer's paper, the object appears by the title, viz., he considers in what cases a quartic surface has upon it a system of conics; or, what is the same thing, in what cases there is a system of planes each intersecting the surface in two conics. It is, in the first place, remarked that there is no proper quartic surface cut by every plane in a pair of conics, or even a proper quartic surface cut in a pair of conics by every plane through a fixed point. The cases considered are-I., where the planes are non-tangent planes; II., where they are single tangent planes; and III., where they are double tangent planes. The case I. is-(1) when there is a nodal conic and two cnicnodes; viz., any plane through the 2 cnicnodes gives a section with 4 nodes, therefore a pair of conics, (and the special case of 4 cnicnodes is noticed

1 The Author exhibited, and pointed out some of the properties of, a model of Steiner's surface. 
incidentally);-(2) when there is a nodal line; any plane through the nodal line besides meets the surface in a conic;-(3) when the surface has two "Selbstberïhrungspuncte"; viz., either of these is a point where the tangent plane is replaced by two coincident planes, and which, when a plane passes through it, gives in the section a tacnode, $=2$ nodes; the section by a plane through two such points, consists of two conics touching each other at the point in question: the equation of such a surface is $\phi^{2}=(*)(p, q)^{4}$, where $\phi$ is a quadric function, and $p, q$ linear functions of the coordinates. In all the cases the planes pass through a fixed line, and the surface may be considered as the locus of a variable conic, the plane of which always passes through such line. II. is-(1) Steiner's surface, where every tangent plane meets the surface in a pair of conics; and (2) surface with a nodal conic and one cnicnode, where every tangent plane through the cnicnode meets the surface in a pair of conics. And III. is (1) the surface with a nodal conic, where every double tangent plane meets the surface in a pair of conics: it is shown that there are 5 quadric cones, such that a tangent plane of any one of these cones is always a double tangent plane of the surface. Or the surface is (2) a quartic scroll; any plane through two intersecting lines of the surface besides meets the surface in a conic.

It is in the paper, Cayley (6), remarked, that the quartic surface $(* \gamma U, V, W)^{2}=0$ can also be expressed in the form $U W-V^{2}=0$; under which form the surface is seen to be the envelope of the series of quadric surfaces $(U, V, W \gamma \theta, 1)^{2}=0$; and by reason of this property it is very easy to find the equations of the reciprocal surfaces, or plane-equations of the quartic surfaces in question. And, in the same paper, it is noticed that the surfaces of whe form in question include the reciprocals of several interesting surfaces of the orders $6,8,9,10$, and 12 ; viz., order 6 , parabolic ring: order 8 , elliptic ring: order 9 , centro-surface of paraboloid: order 10, parallel surface of paraboloid; envelope of planes through the points of an ellipsoid at right angles to the radius vectors from the centre: order 12, centro-surface of ellipsoid; parallel surface of ellipsoid.

It will be noticed that several of the papers by Clebsch and others refer in their titles to the "Abbildung" of a surface; viz., they show that a $(1,1)$ correspondence exists between the points of the surface and the points of a plane. The most simple instance is the quadric surface; here, taking any fixed point $O$ on the surface, the line $O P$ drawn to any point $P$ on the surface meets a plane in a point $P^{\prime}$, and the points $P, P^{\prime}$ have, it is clear, a $(1,1)$ correspondence. And, of course, to any curve on the quadric surface there corresponds a curve on the plane, and the discussion of the nature of the plane curves which correspond to the different curves on the quadric surface would constitute a theory of the Abbildung of the quadric surface.

Similarly, as remarked, Clebsch (2), for a cubic surface, taking upon it any two lines which do not meet, if from a point $P$ on the surface we draw, meeting each of the two lines, a line to meet the plane in $P^{\prime}$, then the point $P$ on the cubic surface and the point $P^{\prime}$ on the plane will have a $(1,1)$ correspondence; and we have thus a like theory for the cubic surface. The Abbildung of a cubic surface had been, however, previously effected by Clebsch in the paper "Die Geometrie auf den Flächen 
dritter Ordnung," Crelle, t. Lxv. (1866), pp. 359-380\}, and by Cremona, in a different and really the most simple manner $\left({ }^{1}\right)$, but having a less obvious geometrical signification.

For surfaces of the higher orders, it is only certain surfaces which admit of an Abbildung, or $(1,1)$ correspondence of the points thereof with the points of a plane; viz. (in the same way as a plane curve, in order to its being unicursal, must have a sufficient number of nodes or cusps) a surface, in order that it may thus correspond with the plane (or say, in order that it may be unicursal), must have a sufficient singularity in the way of a nodal or cuspidal curve. The quartic and quintic surfaces considered in the enumerated memoirs are there considered for the sake of the Abbildung theory which they give rise to; whereas, in the present sketch, the Abbildung theory is considered only for the sake of the quartic and quintic surfaces to which the theory has been applied. But the methods of the theory furnish results in relation to these surfaces; and it is proper to give some account of them.

Clebsch's memoirs (2) and (3) relate to the same subject, which is elaborately treated in the latter of them: the former of them contains, however, some valuable remarks which are not reproduced in the other. In these memoirs (2) and (3), after explaining the above method of the transformation of a cubic surface by means of two of the lines thereof, the author goes on to notice that the like method is applicable to certain quartic and quintic surfaces; viz., (1) quartic surface with a nodal conic: there are here, as already mentioned, 16 lines, each meeting the conic; if, selecting any one of these, from a point $P$ on the surface we draw, meeting the line and the conic, a line to cut the plane in $P^{\prime}$, then the points $P$ on the surface and $P^{\prime}$ on the plane have a $(1,1)$ correspondence. (2) Quartic surface with a nodal line: as already mentioned, there are on the surface 128 conics, each meeting the nodal line; selecting any one of these, if from a point $P$ of the surface we draw, meeting the nodal line and the conic, a line cutting the plane in $P^{\prime}$, then the point $P$ on the surface, and the point $P^{\prime}$ on the plane, have a $(1,1)$ correspondence.

Similarly, (3), for a quintic surface having a nodal skew cubic; then if from a point $P$ on the surface we draw, meeting the skew cubic twice, a line to cut the plane in $P^{\prime}$, the point $P$ on the surface and the point $P^{\prime}$ on the plane have a $(1,1)$ correspondence. The nodal skew cubic may break up into a conic and line which meets it, or into three lines, two of them not meeting each other, but each met by the third line; and the like theory applies to these quintic surfaces.

It is to be noticed that (as for the cubic surface) the above methods of Abbildung, although they have the most obvious geometrical significations, are (as explained in the foregoing foot-note) not the most simple ones; but for each of the foregoing cases (1), (2), (3), the most simple transformation is established in the memoirs now under consideration. The memoir [Memoirs (1) and (2)] of Korndörfer, as indicated by its title, relates to the Abbildung of a quartic surface having a nodal conic and 1, 2, 3, or 4 cnicnodes.

1 Any method of transformation leads to an expression of the coordinates of a point on the surface as proportional to rational and integral functions of a given degree $\nu$ of the coordinates $(x, y, z)$ of a point on the plane, and that transformation is the more simple for which $\nu$ has the smaller value: for the method of the text, the value is $\nu=3$, but for the methods previously given by Clebsch and Cremona, it is $\nu=2$.

C. VII. 
Clebsch's paper (4) relates to the Abbildung of a quartic scroll.

As regards quintic surfaces (not being scrolls), we have, so far as I am aware, only the before-mentioned paper, Clebsch (3), relating to quintic surfaces with a nodal skew cubic; and the paper, Clebsch (5), which relates to the Abbildung of a quintic surface having a nodal quadriquadric. The method employed is that of a preliminary Abbildung upon a twofold plane (2-blättrige Ebene); that is, it consists in establishing, in the first instance, a $(1,2)$ correspondence between the surface and the plane; and by means hereof it is shown that there exist on the surface the conics $K$ and $C$ presently referred to, and which give, ultimately, an ordinary Abbildung or $(1,1)$ correspondence of the points of the surface with those of the plane; viz., this final result is as follows:

There is on the surface a system of conics $K$, such that their planes pass through a point and envelope a quadricone; and also 64 conics $C$ each meeting each of the conics $K$ in a single point; we select one of these and call it the conic $C$.

Take now the plane $B_{1}$ of a conic $K_{1}$ of the series of conics $K$, which plane $B_{1}$ passes, of course, through the vertex $V$ of the cone enveloped by the planes of the conics $K$; viz., these planes intersect the plane $B_{1}$ in a series of lines passing through the point $V$.

Take a point $P$ on the surface; this lies on a conic $K$ meeting the conic $C$ in a point $\xi$; and if we draw the line $\xi P$ to meet the plane $B_{1}$ in $P^{\prime}$, then $P$ on the quintic surface, and $P^{\prime}$ on the f.ane $B_{1}$, will have a $(1,1)$ correspondence; in fact, it appears that, given $P$, there exists a single position of $P^{\prime}$; and conversely, given $P^{\prime}$, this lies on a line $V P^{\prime}$ through which there passes the plane $B_{1}$ and one other tangent plane of the cone: this tangent plane contains a conic $K$ meeting the conic $C$ in a point $\xi$; and joining $\xi P^{\prime}$, this meets the conic $K$ in one other point $P$; viz., given $P^{\prime}$, there is a single position of $P$; and there is thus a $(1,1)$ correspondence.

There are, as originally shown by Schläfli, and as further appears by my memoir on cubic surfaces, Cayley (5), 3 kinds of cubic surfaces of the class 5 ; viz., these are the surfaces $\mathrm{XIII}=12-B_{3}-2 C_{2}, \mathrm{XIV}=12-B_{5}-C_{2}$, and $\mathrm{XV}=12-U_{7}$; for each of these the reciprocal surface is a quintic surface of the class 3 , having a nodal line and a cuspidal quartic curve. For the reciprocal of XIII, the cuspidal curve is a quadriquadric; for that of XIV, the cuspidal curve breaks up into the nodal line (viz., this is a cuspnodal line) and into a skew cubic; for that of $\mathrm{XV}$, the cuspidal curve is a cuspidal quadriquadric, or curve of intersection of two quadric surfaces with singular contact.

It only remains to speak of Schwarz's memoir on quintic scrolls: it is to be remarked that the theory of scrolls is allied more closely with that of plane curves than with that of surfaces; viz., considering any plane section of the scroll, the lines of the scroll have, in general, a $(1,1)$ correspondence with the points of the plane section, and the scrolls of any given order are properly arranged according to the deficiency of the plane section. This is what is done by Cremona in the memoir on quartic 
scrolls above referred to; viz., for a quartic scroll the deficiency is either 0 or 1 ; and of the 12 species, there are 10 for which the deficiency is $=0$ (or which are unicursal), and 2 for which the deficiency is $=1$. And this is the principle of classification in Schwarz's memoir; viz., for a quintic scroll the deficiency is $=0,1$, or 2 ; the number of species established being 10,4 , and 1 for these deficiencies respectively.

\section{List of Memoirs.}

Cayley. 1. Sur la surface des ondes. Liouv: t. XI. (1846), pp. 291-296, [47].

2. Sur un cas particulier de la surface du quatrième ordre avec seize points singuliers. Crelle, t. Lxv. (1866), pp. 284-290, [356].

3. Second Memoir on Skew Surfaces, otherwise Scrolls. Phil. Trans., vol. cLIv. (1864), pp. 559-577, [340].

4. Third Memoir on Skew Surfaces, otherwise Scrolls. Phil. Trans., vol. cLIx. (1869), pp. 111-126, [410].

5. Memoir on Cubic Surfaces. Phil. Trans., vol. Clix. (1869), pp. 231-326, [412].

6. On the Quartic Surfaces $(* \gamma U, V, W)^{2}=0$. Quart. Math. Journ. vol. x. (1868), pp. 24-34; and vol. XI. (1870), pp. 15-25, and pp. 111-113.

7. A Memoir on Quartic Surfaces. Proc. Lond. Math. Soc., vol. III. (1870), pp. $19-69,[445]$.

Clebsch. 1. Ueber die Flächen vierter Ordnung welche eine Doppelcurve zweiten Grades besitzen. Crelle, t. LXIX. (1868), pp. 355-358.

2. Intorno alla rappresentazione di superficie algebriche sopra un piano. Atti del R. Ist. Lomb. (12 Nov. 1868), 13 p.

3. Ueber die Abbildung algebraischer Flächen, insbesondere der vierten und fünften Ordnung. Math. Ann., t. I. (1869), pp. 253-316.

4. Ueber die ebene Abbildung der geradlinigen Flächen vierter Ordnung. welche eine Doppelcurve dritten Grades besitzen. Math. Ann., t. II. (1870), pp. $445-466$.

5. Ueber die Abbildung einer Classe von Flächen fünfter Ordnung. Gött. Ablı, t. xv. 64 p.

Cremona. Sulle superficie gobbe di quarto grado. Mem. di Bologna, t. viII. (30 April, 1868), $15 \mathrm{p}$.

De la Gournerie. 1. Mémoire sur la surface engendrée par la révolution d'une conique autour d'une droite située d'une manière quelconque dans l'espace. Jour. de l'Éc. Polyt., t. xxiII. (1863), pp. 1-74.

2. Mémoire sur les lignes spiriques. Liouv., t. xIv. (1869), 92 p. 
Geiser. Ueber die Flächen vierten Grades welche eine Doppelcurve zweiten Grades haben. Crelle, t. Lxx. (1869), pp. 249-257.

Korndörfer. [There was only a general reference; the several memoirs are

1. Die Abbildung einer Fläche vierter Ordnung mit einer Doppelcurve zweiten Grades und einem oder mehreren Knotenpunkten. Math. Ann., t. I. (1869), pp. $592-626$.

2. Fortsetzung dieses Aufsatzes. t. II. (1870), pp. 41-64.

3. Ueber diejenigen Raumcurven deren Coordinaten sich als rationale Functionen eines Parameters darstellen. t. III. (1871), pp. 415-423.

4. Die Abbildung einer Fläche vierter Ordnung mit zwei sich schneidenden Doppelgeraden. t. III. (1871), pp. 496-522.

5. Die Abbildung einer Fläche vierter Ordnung mit einer Doppelcurve zweiten Grades welche aus zwei sich schneidenden unendlich nahen Geraden besteht. t. Iv. (1871), pp. 117-134.]

Kummer. 1. \{Surfaces of the fourth order with sixteen conical points.\} Berl. Monatsb. (1864), pp. 246-260, and 495-499.

2. Ueber die algebraischen Strahlensysteme, insbesondere über die der ersten und zweiten Ordnung. Berl. Abh. (1866), pp. 1-120.

3. Ueber die Flächen vierten Grades auf welchen Schaaren von Kegelschnitten liegen. Berl. Monatsb. (July, 1863). Crelle, t. LxIv. (1864), pp. 66-76.

Maxwell. On the Cyclide. Quart. Math. Journ., t. Ix. (1867), pp. 111-126.

Schwarz. Ueber die geradlinigen Flächen fünften Grades. Crelle, t. LXvII. (1867), pp. $23-57$. 